\title{
Professor Fengjie He's Experience in Treating Polycystic Ovary Syndrome of Kidney Asthenia and Liver Depression
}

\author{
Chuanyu Wang1, Fengjie He \\ ${ }^{1}$ Shaanxi University of Chinese Medicine, Xianyang 712046, Shaanxi Province, China \\ ${ }^{2}$ The Affiliated Hospital of Shaanxi University of Chinese Medicine, Xianyang 712046, Shaanxi Province, China \\ *Corresponding author: Fengjie He, hefengjie2013@163.com
}

\begin{abstract}
Polycystic ovary syndrome is a common reproductive endocrine metabolic disease in clinic. Professor Fengjie He has accumulated rich clinical experience in the process of diagnosis and treatment of the disease. He believes that the key to the pathogenesis of the disease is the dysfunction of the reproductive axis of "kidney - Tiangui - Chongren - uterus," which is based on kidney deficiency and involves the liver and spleen. Clinically, patients with kidney deficiency and liver depression are common. The treatment takes tonifying the kidney and regulating the menstrual cycle as the main method, and the clinical effect is very effective.
\end{abstract}

Keywords: Polycystic ovary syndrome; Tonifying kidney and regulating the menstrual cycle

Publication date: November 2021; Online publication: November 30, 2021

\section{Introduction}

Polycystic ovary syndrome is mainly characterized by irregular menstruation, infertility, hyperandrogenemia, hyperinsulinemia, etc. There is no record of this disease in TCM classics, but according to its clinical manifestations, it can be classified into the categories of "hypomenorrhea," "amenorrhea," "metrorrhagia and metrostaxis," "infertility" and etc. Professor Fengjie He is one of the first ten famous teachers of Shaanxi University of Chinese Medicine. He has been engaged in the clinical, teaching, research and science of gynecology with integrated traditional Chinese and Western medicine for more than 30 years. Professor Fengjie He has accumulated rich clinical experience in the treatment of polycystic ovary syndrome, and his treatment and medication are unique. The author has the honor to follow his tutor and benefit a lot. The tutor's medication characteristics are summarized as follows.

\section{Etiology and pathogenesis of polycystic ovary syndrome}

\subsection{Kidney deficiency}

Professor Fengjie He believes that the etiology and pathogenesis of polycystic ovary syndrome are complex, mainly kidney deficiency, involving liver and spleen. Women's physiological functions are mainly reflected in menstruation, pregnancy, production and breast milk. The arrival and exhaustion of menstruation and whether they can conceive a fetus are closely related to the kidney. Because the kidney is the foundation of congenital, it stores essence, dominates reproduction, and the kidney Qi plays a leading role in menstruation and pregnancy, and the premise of pregnancy is also based on regular menstruation, just as the "Nvke Zhengzong" says: "Men's semen is strong and women's menstrual periods are normal, which is the way to have a child." Women are "blood-based," blood and essence have the same origin, 
essence can turn into blood, and blood can turn into essence, which has become the material basis of menstruation. The kidney is the viscera of water and fire and the house of Yin and Yang. If kidney-Yin is lost, on the one hand, menstruation will not come on time, on the other hand, oocyte will lose nourishment and cannot mature, and polycystic changes will appear in the ovary. If kidney-Yang is deficient and unable to promote the operation of Qi and blood, the ovum cannot be discharged smoothly during ovulation, resulting in anovulation or even rare ovulation ${ }^{[1]}$. It can be seen from the above that if the function of the kidney is dysfunctional, it will eventually lead to the disorder of reproductive function.

\subsection{Liver depression}

Professor Fengjie He believes that liver depression, insomnia and constipation are the three enemies of women, and the formation of polycystic ovary syndrome is mostly related to liver depression. For example, in "Yijin Gangmu; Volume 6; Qiuzimen; Cure Blood Deficiency and Infertility, it is said: "Women have more Qi and more depression, more Qi is fire, and more depression is stagnation. Therefore, the meridians are not good, and all diseases are made, so the way of fertility is blocked."

The liver governs catharsis. The nature likes to regulate and hate depression. If the liver Qi is regulated and the catharsis is normal, the menstrual period is as usual. In addition, the liver stores blood and controls the blood sea. Women use blood and inject blood sea for menstruation. Essence blood is the material basis for the growth and development of follicles. If the liver blood is insufficient, it will cause blood loss, menstruation cannot come on time, and it is difficult for the uterus to absorb essence into pregnancy ${ }^{[2]}$. The liver and kidney are of the same origin and located in lower energizer together. The kidney is in charge of sealing, the liver is in charge of catharsis, the liver and kidney are coordinated, and menstruation is stored and diarrhea regularly. If the mood is unsuccessful, it can block the impulse of Qi and blood, consume the essence and Qi in the kidney, and become the inducement of the disease.

\section{Treatment experience}

Professor Fengjie He believes that the disease is mainly due to kidney deficiency. In recent years, with the change of social concept and life rhythm, women's pressure is increasing, and liver depression has also become one of the main causes of the disease. Therefore, when treating the disease clinically, the tutor not only pays attention to tonifying the kidney and regulating the menstrual cycle, but also pays attention to dispersing stagnated liver Qi to relieve depression, so as to reconcile Qi, Yang and Yin, and then the menstruation is regulation and the children will come.

\subsection{Tonifying the kidney and regulating the menstrual cycle}

Professor Fengjie He believes that different menstrual cycles should adopt different treatments, but nourishing Yin should run through the whole cycle. In the late menstrual period, nourishing Yin and nourishing blood should be the main. In this period, Yin grew and Yang eliminated. That is, the best period for Yin essence and Tiangui breeding. The purpose is to raise Yin in the blood, and raise essence in Yin. It can adopt Buci Cuhuang Decoction made by the tutor, which contains Radix Angelicae Sinensis, radix rehmanniae preparata, Paeonia lactiflora, Rhizoma Ligustici, Semen Cuscutae, Fructus Lycii, Fructus Ligustri Lucidi, Radix Salviae Miltiorrhizae, Radix Codonopsis, Polygonatum Kingianum and Radix Astragali seu Hedysari. In it, Semen Cuscutae, Fructus Ligustri Lucidi and Polygonatum Kingianum can nourish Yin and blood, liver and kidney; Semen Cuscutae is a natural estrogen that promotes endometrial growth; Radix Angelicae Sinensis, Paeonia lactiflora, Rhizoma Ligustici and Radix Salviae Miltiorrhizae invigorate menstruation, so as to nourish without stagnation; Radix Codonopsis and Radix Astragali seu Hedysari nourish Qi and blood; Fructus Lycii nourish Yin and benefits Yang, so that the Yin Yang rises and the source is not exhausted. The whole prescription played a total of nourishing blood to fill essence, 
nourishing kidney qi, so that Tiangui filled, the sea of blood on time full surplus.

During the intermenstrual, Yin turns to Yang becomes the key, which promotes the expulsion of follicles through dense Qi and blood activity. With the method of tonifying kidney and soothing liver, promoting blood circulation and removing blood stasis, the tutor's self-designed Ovulation Promotion Decoction was adopted. This prescription is based on Liuwei Dihuang decoction, adding Rhizoma Curculigins, epimedium and other worm kidney strengthen Yang products, Fructus Lycii, Semen Cuscutae, Polygonatum Kingianum and kidney tonic products, Radix Angelicae Sinensis, Radix Paeoniae Rubra, Flos Carthami, Herba Lycopi and other products promoting blood and eliminating dysmenorrhea to promote follicular development and excretion. In the early menstrual period, it focuses on tonifying the kidney and helping Yang, but also seeking Yang in Yin, replenishing fire in water and maintaining the function of the corpus luteum. The tutor's self-made promoting Huangtiaojing Decoction is adopted. This prescription is based on Yougui Pill + Siwu Decoction, combined with epimedium, Morinda officinalis and Radix Codonopsis to help the growth of Yang Qi. The menstruation period is mainly focused on promoting blood circulation and regulating menstruation. The purpose is to promote the transformation of Double Yang to Yin, so as to drain the menstrual blood that should be discharged, and facilitate the Yin growth movement at the beginning of the new cycle. The tutor's self-made Shugong Zhuyu Decoction is adapted. Radix Angelicae Sinensis, Rhizoma Ligustici, Semen Persicae, Flos Carthami, Leonurus, Fructus Aurantii, artemisia anomala, Scolopendra, Rhizoma Sparganiiare, Rhizoma Curcumae, Radix Bupleuri, Rhizoma Cyperi, Radix Salviae Miltiorrhizae and Herba Lycopi are used to promote regular menstrual excretion, and prepare for the later period.

\subsection{Soothing liver and relieving depression}

The liver stores blood and controls the sea of blood. It governs catharsis. When the liver Qi reaches, the blood is full and the pulse is smooth. When the sea of blood is full on time, the menstruation will be as usual. If the Qi mechanism is not smooth, the menstruation will be disordered and it is difficult to making babies. Clinically, tutors often use Xiaoyao Powder add and subtract to soothe the liver, relieve depression and regulate Qi. For severe liver depression and discomfort of fullness and distention in both rib-sides, Radix Linderae, Fructus Aurantii Immaturus, Radix Aucklandiae and Rhizoma Cyperi can be added to soothe the liver, relieve depression, promote Qi and relieve pain; For the signs of liver depression and fire, paeonia suffruticosa, Fructus Gardeniae and other heat clearing products can be added; If the patients are depressed and depressed, Radix Curcumae can be added to relieve depression; If sleep is affected by liver depression, Flos Albiziae, Semen Ziziphi Spinosae, Radix Polygalae and other soothing products can be added.

\section{Experience}

Clinically, western medicine often uses hormone therapy for the treatment of polycystic ovary syndrome, which needs long-term drug maintenance, and the condition is easy to repeat after drug withdrawal, which seriously affects the physical and mental health of patients. The tutor Professor Fengjie He paid attention to the combination of disease differentiation and syndrome differentiation in the treatment of polycystic ovary syndrome, and adopted different treatment methods, such as tonifying the kidney and Yang in the early stage, tonifying the kidney and soothing the liver as well as promoting blood circulation and removing blood stasis in the interval, nourishing yin and nourishing blood in the later stage, promoting blood circulation and regulating menstruation in the menstrual period, and adding medicines to soothe the liver and relieve depression, so as to adjust the Yin and Yang of Qi and blood, and establish a normal menstrual cycle and finally realize pregnancy. 


\section{Disclosure statement}

The authors declare no conflict of interest.

\section{References}

[1] Yang B, Zhou Y, Zeng W, et al., 2019, Treatment of Polycystic Ovary Syndrome by Deying Dai. Journal of Changchun University of Traditional Chinese Medicine, 35(02): 239-241 + 245.

[2] Gao Y, Wang G, 2019, Professor Guohua Wang's Experience in Treating Infertility with Non-obese Polycystic Ovary Syndrome. Jilin Journal of Traditional Chinese Medicine, 39(06): 717-721. 